\title{
An Indoor Navigation System using Beacons
}

\author{
Shruti Pokale ${ }^{1}$, Shaikh Abdul ${ }^{2}$, Ravi Mohabe ${ }^{3}$, Sourabh Jichkar ${ }^{4}$, Sanjay Ghodke ${ }^{5}$, Dhiraj Devkar ${ }^{6}$ \\ B.E, Computer Science and Engineering (Final Year), MIT Academy of Engineering, Pune, India ${ }^{1,2,3,4}$ \\ Professor, Department of Computer Science, MIT Academy of Engineering, Pune, India ${ }^{5}$ \\ Technical Head, Upperthrust Technologies, Pune, India ${ }^{6}$
}

\begin{abstract}
In today's era of technology, everything is integrated on smart phone. Every common man has a smartphone and carries it everywhere. When people travel to any new place, in order to locate and find path to their destination, they use GPS in their smart phones. But GPS is not precise for small scale and does not work inside the buildings; therefore the need of indoor positioning arises. Our motivation behind the project is to come up with indoor navigation application that can help any new visitor to locate and find path to their destination easily inside the campus. Indoor navigation application uses BLE beacon. BLE beacon allows mobile application to determine their location on a microlocal scale. Beacon and smartphone use Bluetooth Low Energy technology for communication. Since it uses Bluetooth Low Energy for communication it consume very less battery power.
\end{abstract}

Keywords: iBeacon, BLE, Smartphone, Android Application.

\section{INTRODUCTION}

In today's world technology has much influence on mankind. Human life is dependent on technology. People carry their smart phone wherever they go. Today, mobile phones are not just used for making calls and messaging but for many other purposes. Today smart phone and internet have become essential part of human life. Effective utilisation of technology for human needs can make the living style better. Nowadays location awareness is important need of people.

Today Location Based Services (LBS) [1] are being used in different applications. Since location awareness have become important need of people, location based services are largely used in mobile devices. When people go to any new place GPS can be used to locate and find path to their destination. GPS is not precise for small scale. GPS does not work properly inside building therefore the need of indoor positioning arises. For providing the location based services inside the building we have implemented the indoor navigation application in our project.

Indoor navigation system uses BLE beacon [2] for location awareness. Beacon is a simple device which continuously broadcast BLE signal. This signal allow smartphone to determine their location. Beacon uses Bluetooth Low Energy for broadcasting advertising signals which makes it energy efficient.

\section{LITERATURE SURVEY}

Here we will refer to some previous technologies and techniques used in indoor positioning.

Active Badge technique is based on infrared signals [3]. In this system, Active badge is used which emits infrared signals after fixed time intervals and sends a unique identifier to the receivers. These receivers then send the data to central server. The drawback of this technique is the short range of infrared signals. In addition to that, it also requires direct line-of-sight between receivers and the badge.

Another well-known indoor positioning system is using mTag which uses RFID technology [4]. It is used to define certain RFID receivers which are placed at different locations in an area under investigation. Also, mobile device is pinned with a passive RFID tag which is used to determine its location information. But main drawback of using RFID is high deployment cost.

The limitation of high cost system was eliminated by using Wi-Fi systems for indoor positioning. Some of these systems based on Wi-Fi are RADAR, PlaceLab and Herecast. Amongst these RADAR was the one of the earliest system which is based on IEEE 802.11 standard. These systems used different access points to determine position of the mobile device. The problem with this system is that even if one access point is down, then location information will be distorted and it will give inappropriate result.

We have implemented indoor positioning system using BLE beacon. We are using beacon which uses Bluetooth Low Energy for transmitting advertising signals. Therefore system is very energy efficient compared to other techniques such as Wi-Fi and GPS. 
iBeacon is a technology introduce by Apple in 2013[5]. iBeacon is a technology which gives a higher level of location awareness over a short range distance. Since system uses bluetooth low energy, it consume less battery power, which tends to increase the battery life. Bluetooth Low Energy is a personal area network technology used for transmitting data over short distances.

\section{III.METHODOLOGY}

Indoor navigation application help to locate and guide visitors to navigate through the campus using their mobile devices. Bluetooth Low Energy advertising signals from beacons are the base of our indoor navigation system. Beacons are placed at several location inside the campus. Placement of Beacon device is a very important step to ensure that the desired accuracy is achieved in navigation. Beacon constantly broadcast advertising signal with a unique identifier to nearby mobile devices. Mobile application on smartphone will first locate its position using GPS [6] and then use beacons signal for navigation. Mobile devices can approximately determine their distance to the beacon via quality of signal strengths. Beacons transmits advertising signal to smart phones using Bluetooth Low Energy (BLE). This advertising signal gives location information to mobile device. Mobile application can interpret the signals, and trigger an action that is specified in the application. Approximately based on strength of BLE advertising signal received by mobile device distance is estimated. A routing algorithm calculates the optimal path from user location to destination [7]-[8]. The API gives approximate distance to a beacon in meters. In this way, it is possible to determine users location and continuously and navigate them in campus. Mobile application not only help users to know their position but also help them to find route to their destination inside the campus. Thus indoor navigation system can be used to provide better experience to users.

BLE advertising signal consists of three identifying fields which used to unique identify each device.

A. UUID

UUID stands for "Universally Unique Identifier". It is specific to beacon vendor.

B. Major-ID

Different smaller subset of beacons can be identified by using major id. It is specific to a region such as college campus.

\section{Minor-ID}

It is used to uniquely identify individual beacon. It is 16 bit long. It is specific to a subregion, such as department within the college campus.

\section{IV.ARCHITECTURE OF PROJECT}

Given block diagram describe the architecture of the system.

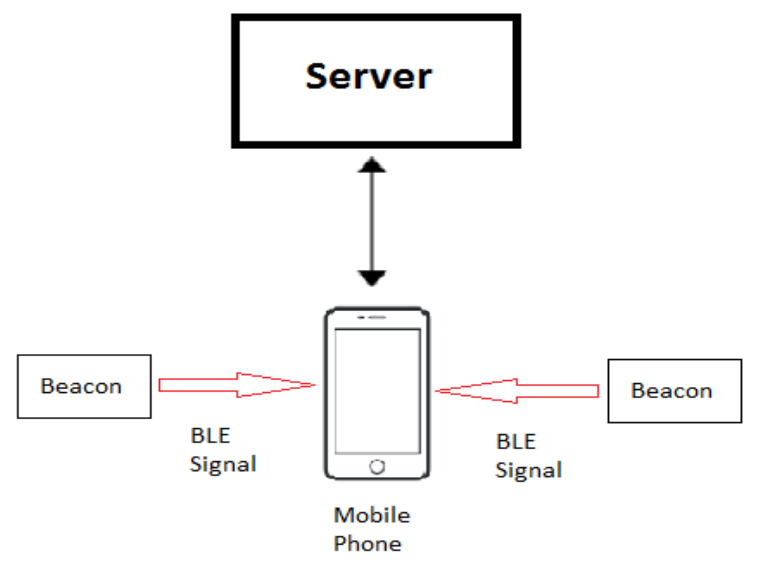

Fig. 1 Architecture of the system

Component of the system are:

A. Android Application

Application recognise BLE advertising signals received from beacons to determine location and finding path to destination. 
B. Beacons

Beacon BLE device which allow mobile devices to understand their position on micro local scale. It uses Bluetooth Low Energy technology.

\section{Bluetooth Low Energy}

It is a wireless personal area network technology used for transmitting data over short distances. Smartphone and beacons communicate using this technology.

\section{CONCLUSION}

Thus providing location based information in mobile application can provide batter experience to user. Location awareness in android application can provide much value to smart phone for better user experience and productivity. In our project we implemented indoor navigation application for college campus. Since application uses Bluetooth Low Energy signals, therefore it consume less battery power. In our project we have implemented more accurate cost efficient approach to indoor navigation.

\section{REFERENCES}

[1] Fard, Hadis Kakanejadi, Yuanzhu Chen, and Kyung Kook Son. ”Indoor Positioning of Mobile Devices with Agile iBeacon Deployment", In Electrical and Computer Engineering (CCECE), 2015 IEEE 28th Canadian Conference on, pp. 275-279. IEEE, 2015.

[2] Moody, Marisa."Analysis of Promising Beacon Technology for Consumers", Elon Journal of Undergraduate Research in Communications 6, no. 1 (2015).

[3] Angel Garcia, Miguel, Carlos Martinez, Jesus Tomas, and Jaime Lloret. "Wireless Sensors self-location in an Indoor WLAN environment.", In Sensor Technologies and Applications, 2007. SensorComm 2007. International Conference on, pp. 146-151. IEEE, 2007.

[4] Korhonen, Jani, TimoOjala, MikkoKlemola, and PasiVaananen. "mTag-Architecture for Discovering Location Specific Mobile Web Services Using RFID and Its Evaluation with Two Case Studies.", In Advanced Int'l Conference on Telecommunications and Int'l Conference on Internet and Web Applications and Services (AICT-ICIW'06), pp. 191-191. IEEE, 2006.

[5] Apple documentation on ibeacon, https://developer.apple.com/ibeacon/getting-started-with-ibeacon.pdf."

[6] Parthasarathy, Jagannathan. "Positioning And Navigation System Using GPS.", International Archives of the Photogrammetry, Remote Sensing and Spatial Information Science 36, no. Part 6 (2006): 208-212.

[7] Srivastava, Rachit, and Anurag Kumar. "Performance analysis of beacon-less IEEE 802.15. 4 multi-hop networks", In 2012 Fourth International Conference on Communication Systems and Networks (COMSNETS 2012), pp. 1-10. IEEE, 2012.

[8] Charfi, Faiza, and Mohamed Bouyahi. "Performance evaluation of beacon enabled IEEE 802.15. 4 under NS2.", arXiv preprint arXiv:1204.1495(2012). 